\title{
Striving for consistent, multidisciplinary, evidence-based counselling for all patients
}

\author{
Francis Lemire, $M D^{\prime}$; Luke T. Lavallée, $M D^{1,2}$ \\ 'Division of Urology, University of Ottawa, Ottawa, ON, Canada; ${ }^{2 T h e}$ Ottawa Hospital Research Institute, University of Ottawa, Ottawa, ON, Canada
}

Cite as: Lemire F, Lavallée LT. Striving for consistent, multidisciplinary, evidence-based counselling for all patients. Can Urol Assoc J 2022;16(2):41. http://dx.doi.org/10.5489/cuaj.7759

\section{See related article on page 35}

I n the study by Earis in this month's issue of CUAJ, patients with a small renal mass (SRM) attending a multidisciplinary clinic, comprised of a urologist and interventional radiologist, made different management choices than a historical cohort of patients primarily managed by a urologist alone. ${ }^{1}$ Notably, almost $50 \%$ of patients in the multidisciplinary clinic cohort selected thermal ablation or irreversible electroporation therapy compared to $12.7 \%$ selecting these treatments historically. Consequently, the rate of active surveillance and surgery decreased for patients seen in the multidisciplinary clinic. Whether these choices led to better long-term outcomes and satisfaction with care is unknown.

As physicians, we should endeavour to provide care that is personalized to the patient's contexts, values, and preferences. A component of this includes providing each patient with evidence-based counselling about all management options. Multidisciplinary clinics may benefit patients by reducing provider biases and diminishing the time and cost of appointments. Previous studies have shown that multidisciplinary discussions frequently lead to changes in management for patients with cancer. ${ }^{2}$ Of note, in this study, patients were directly incorporated in the discussion, whereas many prior studies report the outcomes of physician meetings alone. The authors of this study should be commended for their efforts to improve the patient experience.

While the results of the study are appealing, the authors acknowledge that their comparison to a historical cohort of patients managed from 2012-2016 may have introduced bias. It is likely some changes in management choice between the two cohorts are attributable to change in practice patterns over time. For example, there are now more data supporting outcomes for patients receiving ablative therapies. Moreover, increasing experience with ablation may have provided more comfort and skill, broadening the sample of patients deemed eligible for this treatment. It is also possible that surgical treatment options may influence a patient's decision; for example, access to minimally invasive partial nephrectomy may render surgical management more appealing to some.

Care equity is also important when advocating for multidisciplinary clinics. While multidisciplinary clinics aim to improve equity, access to such clinics may be challenging in Canada due to the immense geographic landscape and differences in availability of local experts. For example, many hospitals have urologists managing SRM but don't have radiologists performing ablative therapies. The rapid adoption of virtual care resulting from the COVID-19 pandemic may help alleviate some of these limitations. Patient decision aids can also help urologists facilitate personalized care. Applicable to this study, McAlpine et al developed a tool for patients with a SRM considering active surveillance, thermal ablation, partial and radical nephrectomy. ${ }^{3}$ Urologists who don't have access to a multidisciplinary setup like the one reported in this study could consider incorporating decision aids into their practice.

Competing interests: The authors do not report any competing personal or financial interests related to this work.

\section{References}

1. Earis D, Wall C, Sinclair N, et al. The impact of a multidisciplinary small renal mass clinic on patient treatment decisions. Can Urol Assoc J 2022;16:35-40. https://doi.org/10.5489/cuai.7307

2. Kočo L, Weekenstroo HHA, Lambregts DMJ, et al. The effects of multidisciplinary team meetings on clinical practice for colorectal, lung, prostate, and breast cancer: A systematic review. Cancers 2021;13:4159. https://doi.org/10.3390/cancers13164159

3. McAlpine K, Breau RH, Stacey D, et al. Shared decision-making for the management of small renal masses - development and acceptability testing of a novel patient decision aid. Can Urol Assoc J 2020;14:385-391. https://doi.org/10.5489/cuai.6575

Correspondence: Dr. Luke T. Lavallée, Division of Urology, University of Ottawa, Ottawa, ON, Canada; lulavallee@toh.ca 